\title{
DIELECTRIC RELAXATION PROCESSES IN SMOKY QUARTZ CRYSTALS AT VERY LOW TEMPERATURES
}

\author{
W. J.DE VOS and J. VOLGER \\ Fysisch Laboratorium der Rijksuniversiteit, Utrecht, Nederland
}

Received 4 April 1967

\begin{abstract}
The relaxation time governing the dielectric loss of smoky quartz crystals appears to level off as a function of temperature below $12^{\circ} \mathrm{K}$, approaching a value of about $1 \mathrm{msec}$. The relaxational behaviour of the colour centres is discussed in terms of tunnelling processes.
\end{abstract}

Under favorite conditions, lattice imperfections at which electrons are trapped (e.g. colour centres) may exhibit a Debye type dielectric response $[1,2]$. A very clear case is found with smoky quartz crystals [3], which has recently been reinvestigated [4] by the present authors, especially between $20^{\circ}$ and $160^{\circ} \mathrm{K}$. In this letter we wish to report our measurements on the temperature dependence of the dielectric relaxation time extended downwards to $4^{\circ} \mathrm{K}$, using the same specimen, with $E / /$-axis.

According to the well-known Debye relaxation theory the dielectric loss factor $\operatorname{tg} \delta$ as a function of frequency and temperature should follow the expression

$$
\operatorname{tg} \delta=\frac{4 \pi}{3}\left(\frac{\epsilon+2}{3}\right)^{2} \frac{N p^{2}}{k T} \frac{\omega \tau}{1+\omega^{2} \tau^{2}}
$$

in which $\epsilon$ is the dielectric constant of the matrix, $N$ and $p$ the concentration and dipole strength of the centres, and $T$ the absolute temperature.

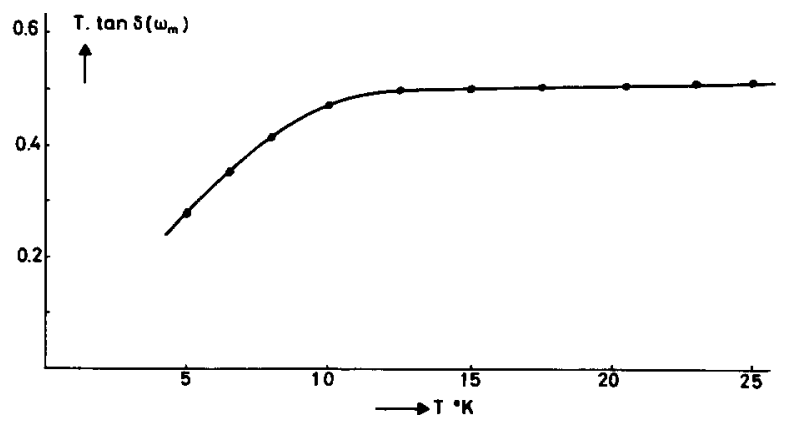

Fig. 1. $T \cdot \operatorname{tg} \delta\left(\omega_{\max }\right)$ as a function of temperature, for a smoky quartz crystal, $\operatorname{tg} \delta(\omega)$ being maximum at $\omega_{\max }$.
Our measurements indicate that the frequency dependence of $\delta$ is in accordance with (1). However, as may be seen from fig. $1, T \cdot \operatorname{tg} \delta\left(\omega_{\max }\right)$ decreases strongly with decreasing temperature, particularly below $10^{\circ} \mathrm{K}$, indicating that $p$ is probably a temperature dependent quantity. The derivation of $\tau$ values from $\operatorname{tg} \delta$ versus $T$ curves taken at constant $\omega$, which procedure is often regarded as useful and rather reliable, will therefore give wrong results in this case. This may explain why our results differ from those of Taylor and Farnell, who reported similar experiments as part of their work on spin lattice interaction in colour centres in quartz [5].

Fig. 2 shows $\ln \tau$ as a function of $1000 / T$ over the temperature range from $100^{\circ}$ to $4^{\circ} \mathrm{K}$. It may be concluded that $\tau$ reaches at low temperature a limiting value of $1.4( \pm 0.1) \mathrm{msec}$. The behaviour of $\tau$ can be described with

$$
1 / \tau=1 / \tau_{1}+1 / \tau_{2}+1 / \tau_{\text {ph }}
$$

with $\quad \tau_{i}=\tau_{0} i \exp \left(E_{i} / k T\right), \quad i=1,2$

and $\tau_{\text {ph }}$ nearly cons tant with respect to $T$. The numerical values are

$$
\begin{array}{rlrl}
\tau_{01} & =(3.0 \pm 0.5) \times 10^{-7} \mathrm{~s} & & E_{1}=8.5 \pm 0.5 \mathrm{meV} \\
\tau_{02} & =(2.5 \pm 1) \times 10^{-11_{\mathrm{S}}} & E_{2}=85 \pm 5 \mathrm{meV} \\
\tau_{\mathrm{ph}}=\text { about } 10^{-3} \mathrm{~s} & & &
\end{array}
$$

An explanation of this relaxational behaviour can be given in terms of a tunneling process by which the electron, trapped near a defect, may pass through potential barriers between those equivalent sites at which the electron may be localized more specifically. Thermal excitation may then bring the electron also from the ground state to (in the case of smoky quartz at least 2) intermediate states in which phonon-assisted transitions through the potential barrier may occur $[4,6,7]$. 


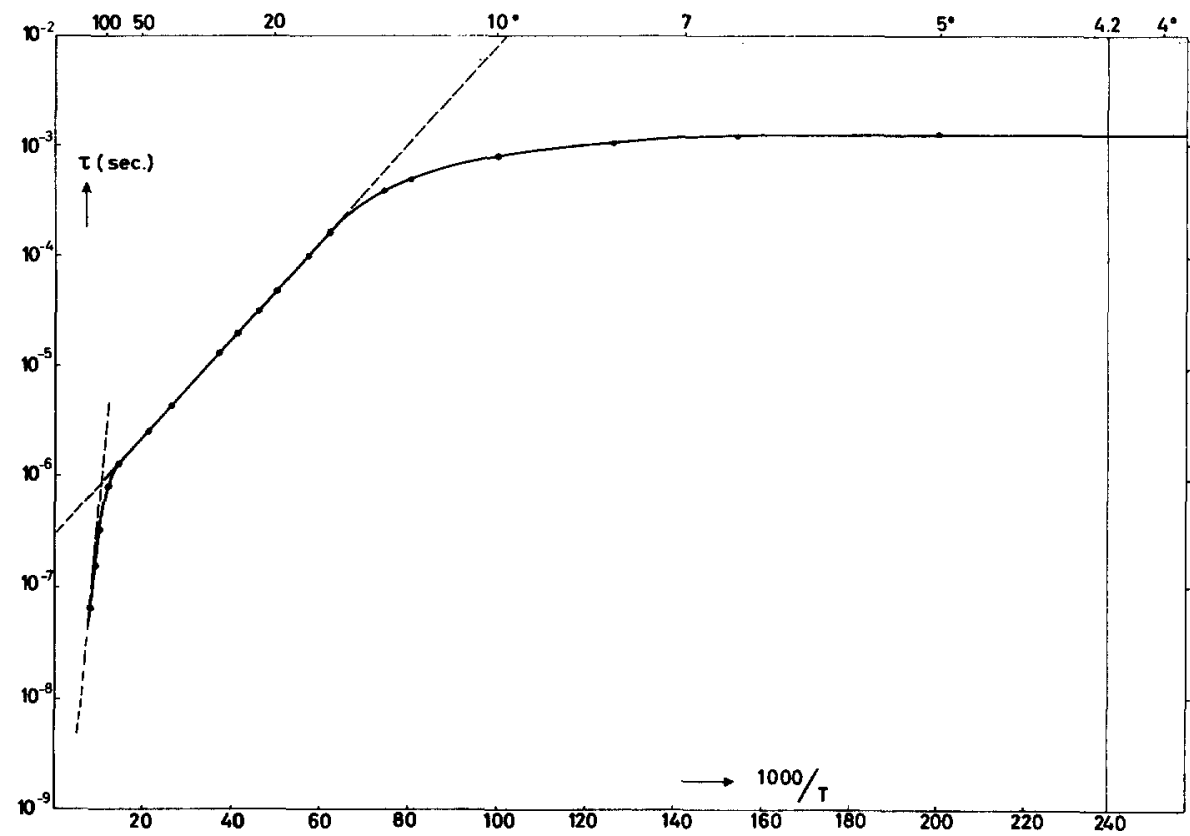

Fig. 2. The relaxation time $\tau$, as determined from dielectric loss measurements, plotted logarithmically as a function of $1 / T$, for a smoky quartz crystal.

The energy levels of these excited states obviously are 8.5 and $85 \mathrm{meV}$ above ground level. However, the tunnelling transition probability in the ground state itself is apparently dominant below $12^{\circ} \mathrm{K}$, and depends hardly on temperature. The temperature dependence of $\tau_{\mathrm{ph}}$ seems to be less than predicted by Sussmann.

The authors gratefully acknowledge the hospitality of Philips Research Laboratories, Eindhoven, Nederland, where some measurements at helium temperatures could be done, and the help of M.M.Laudy and Knol.

\section{References}

1. J. Volger, Discussions Faraday Soc. 23 (1957) 63.

2. J. Volger, Progress in Semi-conductors 4 (1959) 205

3. J.M.Stevels and J.Volger, Philips Research Reports 17 (1962) 283.

4. W. J.de Vos and J. Volger, Physica, to be published.

5. A. L.Taylor and G.W.Farnell, Can.J.Phys. 42 (1964) 595.

6. H. Fröhlich, S. Machlup and T.K. Mitra, Phys. kondens, Materie 1 (1963) 359.

7. J.A.Sussmann, Phys. kondens. Materie 2 (1964) 146. 